\title{
As competências da psicopedagogia nos processos de ensino-aprendizagem no setor de recursos humanos das cooperativas de crédito
}

\section{The competences of psychopedagogy in teaching-learning processes in the human resources sector of credit cooperatives}

\author{
DOI: $10.46814 /$ lajdv2n2-002
}

Recebimento dos originais: 01/02/2020

Aceitação para publicação: 10/02/2020

\section{Jaciara Xavier Pereira Ribeiro}

Doutoranda em Ciências da Educação pela Instituição Brasil de Educação e Pesquisa - IBEP, em parceria com a Universidade Nacional de La Plata e Universidade Del Sol

Instituição: Instituição Brasil de Educação e Pesquisa - IBEP, em parceria com a Universidade Nacional de La Plata e Universidade Del Sol - UNADES/IBEP/BRASIL

Endereço: UNADES - 14 De Mayo 462, Asunción, Paraguai/ IBEP - sede no Calçadão Arthur Bernardes, $n^{\circ}$ 152, Sala 06, Bairro Centro, Viçosa - MG - CEP 36.570- 061

E-mail: jaciaraxp@yahoo.com.br

\section{RESUMO}

A Psicopedagogia é um campo de estudo recentes, principalmente no Brasil tendo como desafio a construção da identidade do Psicopedagogo seu campo de atuação, vivenciados no cotidiano do processo de ensino- aprendizagem. Sendo assim, para a definição do problema de pesquisa levou-se em consideração a expectativa de investigar a importância da Psicopedagogia nos processos de EnsinoAprendizagem. Tendo como objetivo geral: Apresentar conceitos importantes na área da Psicopedagogia nos processos de Ensino-aprendizagem e os objetivos específicos são: demonstrar conceitos sobre a Psicopedagogia; descrever a Psicopedagogia como uma junção da Pedagogia, Psicologia e outras Ciências; apresentar o profissional Psicopedagogo e por fim, descrever a importância do psicopedagogo no setor de Recursos Humanos das Cooperativas de Crédito. Os objetivos foram alcançados, respondendo à questão problema. Como limitação do artigo, a metodologia utilizada para pesquisa foi feita apenas revisão documental e bibliográfica, neste sentido, sugere-se a realização de novas pesquisas, desta vez de forma quantitativa ou qualitativa em ambientes escolares e também em Instituições financeiras, a exemplo das Cooperativas de Crédito, avaliando a importância da Psicopedagogia nos processos de seleção, recrutamento e integração do contratado e na gestão do conhecimento dos profissionais, na busca pelo eficiência e eficácia da organização no mercado de atuação.

Palavras-chave: Psicopedagogia, Psicopedagogo, Pedagogia e Psicologia.

\begin{abstract}
Psychopedagogy is a recent field of study, mainly in Brazil, with the challenge of building the identity of the Psychopedagogue its field of action, experienced in the daily teaching-learning process. Thus, in order to define the research problem, the expectation of investigating the importance of Psychopedagogy in the Teaching-Learning processes was taken into account. Having as general objective: To present important concepts in the area of Psychopedagogy in the teaching-learning processes and the specific objectives are: to demonstrate concepts about Psychopedagogy; describe Psychopedagogy as a combination of Pedagogy, Psychology and other Sciences; introduce the professional Psychopedagogue and, finally, describe the importance of psychopedagogue in the Human Resources sector of Credit Unions. The objectives were achieved, answering the problem question. As a limitation of the article, the methodology
\end{abstract}


used for research was made only documentary and bibliographic review, in this sense, it is suggested to carry out new research, this time in a quantitative or qualitative way in school environments and also in financial institutions, such as the Cooperatives of Credit, evaluating the importance of Psychopedagogy in the selection, recruitment and integration processes of the contractor and in the management of the professionals' knowledge, in the search for efficiency and effectiveness of the organization in the market.

Keywords: Psychopedagogy, Psychopedagogue, Pedagogy and Psychology.

\section{INTRODUÇÃO}

A Psicopedagogia é um campo de estudo recentes, principalmente no Brasil tendo como desafio a construção da identidade do Psicopedagogo seu campo de atuação, vivenciados no cotidiano do processo de ensino- aprendizagem, propondo especialmente alternativas didático-metodológicas que visem contribuir para a redução dos altos índices de fracasso escolar e exclusão social, de forma a se tornar um profissional respeitado pelas instituições, para desenvolvimento do conhecimento.

$\mathrm{O}$ artigo trata sobre a Psicopedagogia que é uma área que estuda e lida com o processo de aprendizagem e com os problemas dele decorrentes, recorrendo aos conhecimentos de várias ciências, sem perder de vista o fato educativo, nas suas articulações sociais mais amplas (SCOZ, 1994, p.12).

A história da psicopedagogia tem início na Europa, em 1946, onde foram fundados os primeiros centros psicopedagógicos por J. Boutonier e George Mauco, com direção médica e pedagógica. Unindo conhecimentos da área de Psicologia, Psicanálise e Pedagogia, esses centros tentavam readaptar crianças com comportamentos socialmente inadequados na escola ou no lar e atender crianças com dificuldades de aprendizagem apesar de serem inteligentes (BOSSA, 2000, p. 39).

Weiss (2001) ressalta, em seus estudos, que a Psicopedagogia é um caminho fundamental à ampliação das possibilidades de busca de qualidade nos processos relacionais, presentes na aprendizagem humana, que ocorre no movimento do desejo, potencialidade maior de cada um de nós, enquanto sujeitos humanos, de melhor construirmos nossas próprias aprendizagens.

O psicopedagogo, no papel de agente corretor, de acordo com Visca (1987), deve priorizar o "conhecimento" do paciente, mesmo que para tal, tenha de realizar encaminhamentos a outros profissionais. O psicopedagogo deverá ter um embasamento teórico para o desenvolvimento de sua função (PERES, OLIVEIRA, 2007).

(...) o objeto de estudo da Psicopedagogia deve ser entendido a partir de dois enfoques: preventivo e terapêutico. O enfoque considera o objeto de estudo da Psicopedagogia o ser humano em desenvolvimento, enquanto educável. Seu objeto de estudo é a pessoa a ser educada, seus processos de desenvolvimento e as alterações de tais processos. Focaliza as possibilidades do aprender, num sentido amplo. Não deve se restringir a uma só agência como a escola, mas ir também à família e à comunidade. Poderá esclarecer, de forma mais ou menos sistemática, a professores, pais e administradores sobre as 
características das diferentes etapas do desenvolvimento, sobre o progresso nos processos de aprendizagem, sobre as condições psicodinâmicas da aprendizagem, sobre as condições determinantes de dificuldades de aprendizagem. O enfoque terapêutico considera o objeto de estudo da psicopedagogia a identificação, análise, elaboração de uma metodologia de diagnóstico e tratamento das dificuldades de aprendizagem (GOLBERT apud BOSSA, 2000, p. 20).

A Psicopedagogia inicialmente foi utilizada como adjetivo, indicando uma forma de atuação que apontava a inevitável interseção dos campos do conhecimento da Psicologia e da Pedagogia (PORTO, 2009).

Sendo assim, para a definição do problema de pesquisa levou-se em consideração a expectativa de investigar a importância da Psicopedagogia nos processos de Ensino-Aprendizagem. O problema é expresso, então, pela questão: quais as competências da Psicopedagogia nos processos de EnsinoAprendizagem? Tendo como objetivo geral: Apresentar conceitos importantes na área da Psicopedagogia nos processos de Ensino-aprendizagem e os objetivos específicos são: demonstrar conceitos sobre a Psicopedagogia; descrever a Psicopedagogia como uma junção da Pedagogia, Psicologia e outras Ciências; apresentar o profissional Psicopedagogo e por fim, descrever a importância do psicopedagogo no setor de Recursos Humanos das Cooperativas de Crédito, que são instituição financeira participantes do mercado financeiro, que investem a capacitação profissional de seus colaboradores.

Vergara (2005, p.32) aborda que o autor de um estudo o justifica ao atribuir-lhe contribuições de ordem prática ou ao estado da arte na área. Neste sentido o presente trabalho poderá trazer como contribuição prática, o conhecimento sobre a Psicopedagogia.

A METODOLOGIA utilizada quanto aos meios de investigação, para a realização deste estudo, tornou-se necessário o desenvolvimento de uma pesquisa documental e de caráter bibliográfica, conceituando termos relacionados aos conceitos gerais sobre Psicopedagogia.

Este artigo está estruturado em capítulos, incluindo a introdução, na qual se apresenta o problema de pesquisa, os objetivos, a justificativa, metodologia e a estrutura do artigo. Os demais capítulos organizam-se da seguinte maneira: no segundo aborda-se sobre conceitos sobre a psicopedagogia, Psicopedagogia como uma junção da Pedagogia, Psicologia e outras ciências; no terceiro formulam-se as conclusões, acrescidas das limitações e sugestões para novos estudos, e por fim são apresentadas as referências bibliográficas.

\section{DESENVOLVIMENTO}

A Psicopedagogia é um campo de estudo recentes, principalmente no Brasil tendo como desafio a construção da identidade do Psicopedagogo seu campo de atuação, vivenciados no cotidiano do processo de ensino- aprendizagem, propondo especialmente alternativas didático-metodológicas que 
visem contribuir para a redução dos altos índices de fracasso escolar e exclusão social, de forma a se tornar um profissional respeitado pelas instituições, para desenvolvimento do conhecimento.

Aranha (2000), que menciona o principal valor que permeia, portanto, a ideia da inclusão é o configurado no princípio da igualdade, pilar fundamental de uma sociedade democrática e justa: a diversidade requer a peculiaridade de tratamentos, para que não se transforme em desigualdade social. A Psicopedagogia contribui para inclusão social, por visar solucionar os problemas no indivíduo que necessita de apoio.

A Psicopedagogia se apresenta com um caráter multidisciplinar devido à complexidade dos problemas de aprendizagem, que busca conhecimento em diversas outras áreas do conhecimento, além da psicologia e da pedagogia. Lidar com o insucesso escolar, com o baixo rendimento, com as múltiplas implicações para a autoavaliação da criança, para a família, professores e comunidade constitui-se em tarefa complexa e desafiadora para a qual não se tem ainda uma resposta acabada e pronta, o que aponta para a necessidade de buscar alternativas que possam minimizar tal situação (OKANO et al, 2003).

Alicia Fernández (2001) destaca que é preciso, no campo psicopedagógico, sempre incorporar novos saberes e conhecimentos sobre a inteligência, o corpo, o desejo e o organismo pelo fato de que estes são os principais níveis imbricados no ato humano de aprender: cada sujeito, em seus processos, possui sua própria modalidade de aprendizagem, o que quer dizer que cada um, em sua individualidade, possui suas próprias condições, seus limites e meios para acessar conhecimentos e construir saberes. No que diz respeito ao produzir conhecimento em Psicopedagogia, é também importante ampliar o ambiente e a atuação daquele profissional que pesquisa: de modo geral, é preciso perceber que a pesquisa só pode ser considerada centro de seu aspecto educativo, ou seja, da própria formação do psicopedagogo.

Para obtermos algum domínio de nossos processos de autoria de pensamento, é de extrema valia conquistar e exercitar a qualidade de sermos produtivos, conscientes e emancipados, tornando-nos sujeitos capazes de encontrar nossos próprios espaços e tempos, e sermos desejantes de crescimento, recusando-nos cotidianamente a sermos apenas objetos, a sermos apenas meros expectadores de todo este movimento.

O saber psicopedagógico se obtém a partir de duas vertentes: da experiência, "mergulhando na tarefa", e através do tratamento psicopedagógico didático. Posicionando-se como observador ou como juiz é muito difícil contatar com o saber. Um espaço importante de gestação do saber psicopedagógico é o trabalho de autoanálise das próprias dificuldades e possibilidades no aprender, pois a formação do psicopedagogo, assim como requer a transmissão de conhecimentos e teorias, também requer um espaço para a construção de um olhar e uma escuta psicopedagógicos a partir de uma análise de seu próprio aprender. 
Dentro dessa conotação adjetiva da psicopedagogia, alguns autores, principalmente pertencentes ao campo pedagógico, no final da década de 70 e início dos anos 80 no Brasil, chamaram de "atitude psicopedagógica" o que em verdade era um "psicologismo radical". Por isso, tratavam de denunciar a formação dos professores por eles cognominada de psicopedagogia (BOSSA, 2000).

Uma parte importante da contribuição psicopedagógica à educação atual é dada como resposta às demandas que recebemos das escolas. Eis uma razão a mais para que essas respostas se fundamentem em profunda compreensão do que as gera e evitem a precipitação ou a ingenuidade das ações originadas de pouca reflexão. A análise de cada uma dessas demandas de intervenção deve servir igualmente para diferenciar as atuações convenientes daquelas que não podemos nos permitir sem redefini-las previamente (BONALS; GONZÁLEZ, 2008).

A Psicopedagogia como uma área de estudo interdisciplinar está integrada por diversas ciências, como pedagógica, psicológica, fonoaudiológica, entre outras, sempre a serviço do desenvolvimento do processo de ensino e aprendizagem. A psicopedagogia apresenta modalidades de atuação como a clínica e a institucional. A intervenção clínica é a mais antiga e surgiu na fronteira entre a psicologia e a pedagogia, privilegiando o atendimento individual de forma terapêutica. Assim, vemos as escolas encaminhando o aluno com dificuldades de aprendizagem para as clínicas. Estas clínicas geralmente trabalham com uma equipe interdisciplinar composta por Psicopedagogo, psicólogos, fonoaudiólogos, médicos, entre outros, que após o diagnóstico do problema, iniciam o tratamento.

A intervenção institucional é mais recente. Ela geralmente é desenvolvida na própria escola com o objetivo de prevenir ou superar as possíveis dificuldades de aprendizagem.

Desta forma, a Psicopedagogia tem procurado contribuir para a conscientização da importância do ato educativo, através de uma prática transformadora, visando especialmente o sucesso do aluno e a melhoria na qualidade do processo de ensino e aprendizagem (OLIVEIRA, 2001).

Morais (2004) considera a Psicopedagogia Institucional um modelo teórico-prático que permite um questionamento, um diagnóstico e uma elaboração de recursos para a solução de problemas em situações de carência, conflito, crise, em instituições educacionais.

Jorge Visca (1987) compreendeu a Psicopedagogia como uma possibilidade de termos uma visão mais ampliada dos complexos processos que nos levam à efetiva aprendizagem.

\subsection{A PSICOPEDAGOGIA COMO UMA JUNÇÃO DA PEDAGOGIA, PSICOLOGIA E OUTRAS CIÊNCIAS}

Historicamente, a Psicopedagogia surgiu na fronteira entre a Pedagogia e a Psicologia, a partir das necessidades de atendimento de crianças com "distúrbio de aprendizagem", consideradas inaptas dentro do sistema educacional convencional. (...) No momento atual, à luz de pesquisas psicopedagógicas 
que vêm se desenvolvendo, inclusive no nosso meio, e de contribuições da área da psicologia, sociologia, antropologia, linguística, epistemologia, o campo da psicopedagogia passa por uma reformulação. De uma perspectiva puramente clínica e individual, busca-se uma compreensão mais integradora do fenômeno da aprendizagem e uma atuação de natureza mais preventiva. (KIGUEL apud BOSSA, 2000, p. 18). Assim, o termo já foi inventado e assinala de forma simples e direta uma das mais profundas e importantes razões da produção de um conhecimento científico, a Psicopedagogia, que nasceu da necessidade de uma melhor compreensão do processo de aprendizagem, não se basta como aplicação da Psicologia à Pedagogia. (...) sendo assim, pode-se defini-lo como aplicação da psicologia experimental à pedagogia. (MACEDO apud BOSSA, 2000, p. 17)

$\mathrm{Na}$ concepção de Polity (2002), a pedagogia com enfoque construtivista com base no Construcionismo social elenca três fatores básicos do processo educacional: a interdisciplinaridade, a interacionalidade e o pensamento complexo conduzindo o educando para a prática da transformação social. A autora faz a relação entre as dificuldades do aluno a as dificuldades do professor no processo ensino-aprendizagem, interrelacionando-os, até mesmo, nos fracassos, Pois a Constituição Federal do Brasil assume como fundamental, dentre outros, o princípio da igualdade, quando reza no caput de seu artigo 5, que "todos são iguais perante a lei, sem distinção de qualquer natureza, garantindo-se aos brasileiros e aos estrangeiros, residentes no País, a inviolabilidade do direito à vida, à liberdade, à igualdade, à segurança e à propriedade”, o mesmo mencionado por Aranha (2000), o principal valor que permeia, portanto, a ideia da inclusão é o configurado no princípio da igualdade, pilar fundamental de uma sociedade democrática e justa: a diversidade requer a peculiaridade de tratamentos, para que não se transforme em desigualdade social.

A Psicopedagogia, como área de aplicação, antecede o status de área de estudos, a qual tem procurado sistematizar um corpo teórico próprio, definir o seu objeto de estudo, delimitar o seu campo de atuação e, para isso, recorre à Psicologia, Psicanálise, Linguística, Fonoaudiologia, Medicina, Pedagogia (PORTO, 2009). Desta forma, falar sobre psicopedagogia é, necessariamente, falar sobre articulação entre educação e psicologia, articulação essa que desafia estudiosos e práticos dessas duas áreas. Embora quase sempre presente no relato de inúmeros trabalhos científicos que tratam principalmente dos problemas ligados à aprendizagem, o termo psicopedagogia não consegue adquirir clareza na sua dimensão conceitual. (NEVES apud BOSSA, 2000, p. 18).

A afirmação de que a Psicopedagogia, historicamente, surgiu na fronteira entre a Psicologia e a Pedagogia merece maior atenção. 
Menciono as tentativas de explicação para o fracasso escolar por outras vias que não a pedagógica e a psicológica. (...) os fatores etiológicos utilizados para explicar índices alarmantes do fracasso escolar envolviam quase que exclusivamente fatores individuais, como desnutrição, problemas neurológicos, psicológicos, etc. (...) No Brasil, particularmente durante a década de 70, foi amplamente difundido o rótulo de Disfunção Cerebral Mínima para as crianças que apresentavam, como sintoma proeminente, distúrbios na escolaridade (KIGUEL apud BOSSA, 2000, pp. 18-19).

Portanto, vemos que Psicopedagogia estuda as características da aprendizagem humana: como se aprende, como essa aprendizagem varia evolutivamente e está condicionada por vários fatores, como se produzem as alterações na aprendizagem, como reconhecê-Ias, tratá-Ias e preveni-Ias (PORTO, 2009).

\subsection{O PSICOPEDAGOGO}

A profissão do psicopedagogo não está regulamentada, mas o projeto se encontra na Comissão de Constituição, Justiça e Redação, na Câmara dos Deputados Federais, para ser aprovada e tem sido oferecido por instituições devidamente autorizadas ou credenciadas, porém tem sido a dificuldade dos psicopedagogos em propor procedimentos de avaliação e de intervenção. Na concepção de Carvalho (2011), as dificuldades muitas vezes são de fatores externos (ambiental), ou seja, estereótipos criados pela família e também pela escola/professores. Portanto, os Psicopedagogos precisam conhecer a causa das dificuldades para encontrar meios de ajudar o aluno e não para excluí-lo.

De acordo com Chamat (2008), seu papel é de focalizar a problemática dentro do contexto causa/sintoma e atuar sobre eles. Deve planejar sua atuação desde o contato telefônico. Este, muitas vezes, fornece dados de como estão as relações vinculares familiares.

Segundo Chamat (1996), após realizar todo o diagnóstico psicopedagógico, o psicopedagogo estará de posse do quadro "patológico" sobre o caso em estudo, bem como de um sistema de hipóteses que lhe permita direcionar o tratamento psicopedagógico.

\subsubsection{O psicopedagogo inserido no setor de Recursos Humanos das Cooperativas de Crédito}

O cooperativismo é tido como um dos principais instrumentos para a promoção de desenvolvimento econômico e social, pois gera e distribui renda e promove o capital social nas comunidades que o praticam (BIALOSKORSKI NETO, 2002, p.77:97).

O cooperativismo está embasado em sete princípios, quais sejam: adesão voluntária e livre, gestão democrática pelos membros, participação econômica dos membros, autonomia e independência, educação, formação e informação, intercooperação, interesse pela comunidade. (STEFANO; ZAMPIER; GRZESZCZESZYN, 2006). Estes princípios veem se modificando ao longo dos tempos. A Organização das Cooperativas Brasileiras (OCB) classifica as cooperativas do Brasil em treze ramos distintos: agropecuário, consumo, crédito, educacional, especial, habitacional, infraestrutura, mineral, produção, 
saúde, trabalho, transporte e turismo e lazer (OCEMG, 2011, p.8:9). Com destaque ao ramo crédito que segundo Pinheiro (2008, p.7), as cooperativas de crédito são instituições financeiras constituídas sob a forma de sociedade cooperativa, tendo por objetivo a prestação de serviços financeiros aos associados.

As cooperativas de crédito vêm crescendo muito no sistema financeiro e tem buscado no mercado, os melhores profissionais, encontrados através de realização de processos de recrutamento e seleção e integração do novo profissional na Instituição, que presta os mesmos serviços financeiros que os bancos, tendo em seus princípios as principais diferenças entre as partes. "o capital humano enquanto elemento detentor da aprendizagem organizacional passa a ser o diferencial, e com ele a necessidade cada vez maior das organizações de aprender e de desenvolver novos conhecimentos (FLEURY \& OLIVEIRA JUNIOR, 2002 apud NASCIMENTO, 2008)”.

\begin{abstract}
Sendo assim, o psicopedagogo torna-se um aliado ao setor de Recursos Humanos, pois sua atuação é de formação, desenvolvimento profissional e treinamento. O psicopedagogo atuará avaliando e controlando a aprendizagem, o que tornará o processo de recrutamento e seleção mais qualificado; fará um levantamento do perfil de cada colaborador da Organização para identificar o nível de conhecimento e se há necessidade de treinamento ou aperfeiçoamento. Atuará também na intervenção para que os colaboradores que resistam às mudanças aceitem da melhor maneira possível, alcançando o desenvolvimento do grupo na Organização. Sabemos que existem diversos tipos de Organizações, sejam elas públicas, privadas, filantrópicas ou do Terceiro Setor, cada uma possui um objetivo, uma característica, mas todas sofreram mudanças devido à economia globalizada. Com tantas mudanças o Mercado de Trabalho passou a exigir cada vez mais das Organizações e como consequência, estas passaram a exigir cada vez mais de seus profissionais. As Organizações e os profissionais precisam se adaptar às mudanças e para isso é preciso aceitálas, pois mudança é questão de sobrevivência (BENGEZEN, 2018).
\end{abstract}

Aprimorar as competências, sejam elas técnicas ou comportamentais dos seus colaboradores, são uma das necessidades das cooperativas de crédito que estão investindo cada vez mais na aprendizagem, pois identificam a importância do aperfeiçoamento ou treinamento para aprimoramento, devido exigência do mercado financeiro e de trabalho, fazendo com que a instituição financeira continue a evoluir sempre de forma eficiente e eficaz. A Era da Tecnologia da Informação aumentou ainda mais a competitividade, surgiu uma nova concepção, uma nova forma de as Cooperativas de crédito atuarem. O capital humano perdeu lugar para o capital intelectual, ou seja, o conhecimento passou a ser o mais importante. A estrutura organizacional não é mais a mesma; optaram por modelos mais inovadores que foram ajustados às mudanças, à modernidade. Surgiu então o Departamento de Recursos Humanos para lidar com essas transformações e promover melhoria (BENGEZEN, 2018). 
Atualmente o empreendedorismo se destaca, o Mercado de Trabalho é mais exigente, a tecnologia está deixando muitos profissionais defasados e necessitando de treinamento ou aperfeiçoamento. As organizações estão preocupadas com a qualidade de vida e com a inteligência emocional dos funcionários, pois a constante mudança precisa ser trabalhada para não ocasionar danos. "Esperar, portanto, que "as pessoas mudem" para promover as mudanças de que a empresa precisa para sobreviver, como é muito comum ouvir-se no ambiente empresarial, é simplesmente suicídio. A empresa pode se acabar antes que se contabilize a primeira "mudança pessoal" Por isso, mudar é preciso, cada ano que passa há mais mudanças e as organizações e profissionais precisam acompanhar e desenvolver métodos para que atendam às exigências do Mercado (BENGEZEN, 2018).

Nas cooperativas de crédito, o psicopedagogo cumpre a importante função de socializar os conhecimentos disponíveis, promover o desenvolvimento cognitivo e a construção de normas de conduta inseridas num mais amplo projeto social, procurando afastar, contrabalançar a necessidade de repressão. Peres e Oliveira (2007) fazem menção com respeito à importância da prevenção e da intervenção psicopedagógica, mas enfatizam também que não podemos ignorar a fase que precede a essas ações. A etapa de avaliar, por exemplo, a avaliação psicopedagógica, deverá anteceder a toda e qualquer proposta de intervenção, seja ela clínica ou institucional, tornando os profissionais mais competentes em suas áreas de atuação.

\section{CONCLUSÃO}

Para a realização deste artigo foi necessária uma revisão documental e bibliográfica sobre o tema abordado, apresentando conceitos sobre a Psicopedagogia. Sendo assim, para a definição do problema de pesquisa levou-se em consideração a expectativa de investigar a importância da Psicopedagogia nos processos de Ensino-Aprendizagem. O problema é expresso, então, pela questão: quais as competências da Psicopedagogia nos processos de Ensino- Aprendizagem? Tendo como objetivo geral: Apresentar conceitos importantes na área da Psicopedagogia nos processos de Ensino-aprendizagem e os objetivos específicos são: demonstrar conceitos sobre a Psicopedagogia; descrever a Psicopedagogia como uma junção da Pedagogia, Psicologia e outras Ciências; apresentar o profissional Psicopedagogo e por fim, descrever a importância do psicopedagogo no setor de Recursos Humanos das Cooperativas de Crédito.

Os objetivos específicos foram alcançados tendo resultados devidamente demonstrado no segundo capítulo deste artigo. O alcance dos objetivos específicos foi favorável ao atendimento do objetivo geral, o qual foi apresentar conceitos importantes na área da Psicopedagogia nos processos de Ensino-aprendizagem sendo respondida a questão problema sendo apresentado no artigo as competências da Psicopedagogia nos processos de Ensino- Aprendizagem.

Diante dos resultados apontados e por tratar-se de um estudo constituído de apenas pesquisa bibliográfica, pôde-se perceber que o artigo traz como limitação, a impossibilidade do levantamento da totalidade das pesquisas realizadas com relação ao tema principal que Psicopedagogia. Neste sentido, 
sugere-se a realização de novas pesquisas, desta vez de forma quantitativa ou qualitativa, por meio de questionários ou entrevista, com vistas a ampliar as análises dos resultados desta pesquisa em ambientes escolares e também em Instituições financeiras, a exemplo das Cooperativas de Crédito, avaliando a importância da Psicopedagogia nos processos de seleção, recrutamento e integração do contratado e na gestão do conhecimento dos profissionais, na busca pelo eficiência e eficácia da organização no mercado de atuação.

\section{REFERÊNCIAS}

ARANHA, Maria Salete F. Inclusão social e municipalização (2000). Disponível em: http://cape.edunet.sp.gov.br/textos/textos/10.doc Acesso em: 21 fev. 2011.

BENGEZEN, Fernanda de Souza. Psicopedagogo inserido no setor de Recursos Humanos https://wwwrhportal.com.br/artigos-rh/psicopedagogo-inserido-no-setor-de-recursos-humanos/ Acessado $28 / 08 / 2018$

BIALOSKORSKI NETO, Sigismundo. Gestão agroindustrial. GEPAI: Grupo de Estudos e Pesquisas Agroindustriais. São Paulo: Atlas, 1997.

BONALS, Joan; GONZÁLEZ, Ângela. A demanda por avaliação psicopedagógica. In: SÁNCHEZCANO, Manuel; BONALS, Joan. Avaliação psicopedagógica. Trad. Fátima Murad. Porto Alegre: Artmed, 2008.

BOSSA, Nadia A. A psicopedagogia no Brasil: contribuições a partir da prática. Porto Alegre: Artes Médicas, 2000.

CARVALHO, Maria. A trajetória da psicopedagogia, suas contribuições e limites. Disponível em: http://www.psicopedagoga.org/index.php?option=com_content\&view=frontpage\&Item id=1 Acesso em: 24 fev. 2011.

CHAMAT, L. S. J. A arte de cultivar. São Paulo: Vetar, 2005.

CHAMAT, L. S. J. Relações vinculares e Aprendizagem. São Paulo: Vetar Editora, 1996.

CHAMAT, Leila Sara José. Técnicas de intervenção psicopedagógica para dificuldades e problemas de aprendizagem. São Paulo: Vetor, 2008.

FERNANDÉZ, Alicia. O saber em jogo: a psicopedagogia possibilitando autorias de pensamento. Porto Alegre: Artmed, 2001.

LEITE, Jacqueline Rosadine de Freitas; SENRA, Ricardo Belízio de Faria. Aspectos Jurídicos das Cooperativas de Crédito. Belo Horizonte: Mandamentos, 2005.

MORAIS, Maria de Lourdes Cysneiros. Bases Conceituais para o Diagnóstico PsicopedagógicoInstitucional (2004). Disponível em:

http://www.abpp.com.br/artigos/51.htm Acesso em: 21 fev. 2011. 
NASCIMENTO, Cláudia Terra do. O Psicopedagogo e a Aprendizagem Organizacional: A Importância da Gestão do conhecimento na Administração de Recursos Humanos. São Paulo, 2008. Disponível em Acesso em: 13 jan. 2010.

ORGANIZAÇÃO E SINDICATO DAS COOPERATIVAS DE MINAS GERAIS. Informações econômicas e sociais do cooperativismo mineiro. Belo Horizonte: Gerência Técnica do Sistema Ocemg/Sescoop-MG. 2011.

OKANO et al. Crianças com dificuldades escolares atendidas em programa de suporte psicopedagógico na escola: avaliação do autoconceito. São Paulo: PRC, 2003.

OLIVEIRA, Antonia Soares Silveira e. Educação inclusiva, utopia possível: uma leitura psicopedagógica de crianças/adolescentes com dificuldades de aprendizagem. Florianópolis: UFSC, 2001. (Dissertação de Mestrado).

PERES, M. R.; OLIVEIRA, M. H. M. A. Psicopedagogia - Limites e possibilidades a partir de relatos de profissionais. São Paulo: PUC, 2007.

PINHEIRO, Marcos Antonio Henriques. Cooperativas de crédito: história da evolução normativa no Brasil.6. ed. Brasília: Banco Central do Brasil, 2008.

POLITY, E. Dificuldade de Ensinagem. São Paulo: Vetor Editora, 2002.

PORTO, Olivia. Bases da Psicopedagogia: diagnóstico e intervenção nos problemas de aprendizagem. 4 ed. Rio de Janeiro: Wak, 2009.

SCOZ, Beatriz. Psicopedagogia e realidade escolar: o problema escolar e de aprendizagem. 2 ed. Petrópolis, RJ: Vozes. 1994.

STEFANO, S.R. ZAMPIER, M. A.; GRZESZCZESZYN, G. Cooperativas: características, gestão e relevância sócio-econômica para o Brasil. In: SEMINÁRIOS EM ADMINISTRAÇÃO - SEMEAD, 9, 2006, São Paulo. Anais... (São Paulo: [s.n.], 2006, 16p.

VERGARA, Sylvia Constant. Projeto e relatórios de pesquisa em administração. São Paulo: Atlas, 2005.

VISCA, Jorge. Clínica Psicopedagógica. Epistemologia convergente. Porto Alegre: Artes Médicas, 1987.

WEISS, Maria Lucia Lemme. Psicopedagogia clínica: uma visão diagnóstica. Porto Alegre: Artes Médicas, 2001. 